\title{
Prevalence and risk factors of hypophosphatemia in pediatric intensive care unit
}

\begin{abstract}
Background: Hypophosphatemia is often unrecognized in critically ill patients despite its potential serious complications.

Objectives: To estimate the prevalence of hypophosphatemia and to identify risk factors and outcome associated with this disturbance in children admitted to PICU.

Subjects and Methods: In a prospective cohort study, 72 children admitted consecutively to a pediatric intensive care unit (PICU) were monitored regarding their phosphorus serum levels during the first 7 days of admission. Age, gender, diagnosis at admission, malnutrition, clinical severity score at admission (pediatric index of mortality or PIM), sepsis (C-reactive protein and cultures), use of dopamine, diuretics, antacids and steroids, starvation period, length of stay and outcome were analyzed as independent variables for hypophosphatemia.
\end{abstract}

\begin{abstract}
Results: More than half of our patients (58\%) had hypophosphatemia on admission and only 5 patients developed hypophosphatemia during their PICU stay. Malnutrition was present in $21 \%$ of patients, but it did not show association with the hypophosphatemia $(\mathrm{p}=0.1753)$. The number of starvation days, PIM, days on mechanical ventilation and survival to discharge were significantly associated with hypophosphatemia $(\mathrm{p}<0.05)$.
\end{abstract}

Conclusion: Hypophosphatemia was a common problem in our PICU and was associated with the presence of respiratory complaints, higher PIM, and increased starvation days. These factors might be considered as risk factors for hypophosphatemia in critically ill children, especially when occur together.

Keywords: critically ill, intensive care unit, malnutrition, phosphorus, starvation days
Volume I Issue 6 - 2014

\author{
Hanaa Ibrahim Rady,' Khalil Abdel,' Khalek \\ Mohamed $^{2}$ \\ 'Department of Pediatrics, Cairo University, Egypt \\ ${ }^{2}$ Pediatrics and Neonatal Intensivist, Egypt
}

Correspondence: Hanaa I Rady, Assistant Professor of Pediatrics, Gameat el Doual Al Arabia Street, Mohandessin, Egypt, Tel +20100 I 482444, Fax 0020233473960,

Email hanaaarady@gmail.com

Received: September 08, 2014 | Published: November 22, 2014
Abbreviations: PICU, pediatric intensive care unit; PIM, pediatric index of mortality; CIC, critically ill children; ESR, erythrocyte sedimentation rate; CRP, c-reactive protein; BUN, blood urea nitrogen

\section{Introduction}

Hypophosphatemia is a metabolic disturbance with potential serious complications and is often unrecognized in critically ill children (CIC). ${ }^{1}$ In a review of clinical studies done on hypophosphatemia in pediatric intensive care unit (PICU) patients, its prevalence exceeded $50 \% .^{2}$ Phosphate ions are critical for normal bone mineralization, and phosphate plays a vital role in a number of other biological processes such as signal transduction, nucleotide metabolism, adenosine triphosphate (ATP) production and enzyme regulation. ${ }^{3}$

Symptoms of hypophosphatemia tend to be nonspecific in the majority of cases and include fatigue and irritability. However, severe hypophosphatemia (less than $1.0 \mathrm{mg} / \mathrm{dl}$ ) may lead to more serious problems ${ }^{4}$ such as reduced diaphragmatic contractility, ${ }^{5}$ cardiac arrhythmias, ${ }^{6}$ myocardial reduction and severe congestive cardiac insufficiency in the postoperative period of cardiac surgery, ${ }^{7}$ leukocyte dysfunction ${ }^{6}$ and neuromuscular disturbances. ${ }^{8}$ Potential risk factors in most patients with hypophosphatemia in the literature include refeeding syndrome, ${ }^{9}$ malnutrition, ${ }^{10}$ starvation for over 3 days, sepsis, Pediatric Index of Mortality (PIM), ${ }^{2,11}$ catecholamine's and antacids, ${ }^{12}$ trauma, diuretic, steroid therapy, ${ }^{2}$ excessive parenteral glucose administration and respiratory alkalosis..$^{13}$

The aim of this study was to estimate the prevalence of hypophosphatemia and to identify risk factors and outcome associated with this disturbance in children admitted to our PICU.

\section{Materials and methods}

\section{Study design and Population}

All infants and children admitted to Cairo University Children Hospital Medical PICU in the period from July 2010 through December 2010 were consecutively and prospectively enrolled excluding those with renal insufficiency. Sample size was determined using power analysis. Power was computed through the statistical package used and the value was 0.896 with alpha level $=0.05$, power $=80 \%$. After calculation, $\mathrm{n}=68$ and we have chosen 72 cases. The study was approved by Cairo University Ethics Committee and was conducted in accordance with the bylaws for human research. The study was explained and consents were obtained from all parents or legal guardians before enrollment. We had 4 drop out patients due to laboratory test availability (absent serum phosphorus level). Drop outs were included in the study and were considered as normophosphatemic patients during the statistical analysis.

\section{Patients were subjected to}

\section{Initial evaluation:}

a. History and clinical examination.

b. Routine laboratory investigations including: complete blood picture, erythrocyte sedimentation rate (ESR), C-reactive protein (CRP), serum electrolytes, serum aminotransferases, blood urea nitrogen $(\mathrm{BUN})$, serum creatinine as well as the appropriate bacterial cultures.

c. Assessment of severity of illness on admission using the PIM. Variables are: Elective admission, specific diagnosis, pupils fixed 
more than $3 \mathrm{~mm}$ in bright light, mechanical ventilation, systolic blood pressure, Base excess, $\mathrm{FIO}_{2}$ and $\mathrm{PCO}_{2}$.

d. The following variables were collected: age, gender, diagnosis, malnutrition (+/- 2 SD weight for height), phosphorus intake if any, clinical severity score at admission (PIM score), sepsis whether clinically suspected (multisystem organ failure, bad general condition) or laboratory proved (+ve culture, +ve CRP, leucocutosis), use of dopamine, frusemide, steroids and starvation period (the period without enteral feeding at the PICU).

e. Special emphasis was out on monitoring serum phosphorus levels during the first 7 days of admission (within first $48 \mathrm{hrs}$ of admission and at day7).

\section{Intervention}

As normal serum phosphorus level varies according to age, we used levels in (Table 1) ${ }^{14}$ as a guide to start phosphorous supplementation to our patients. Since we didn't have patients younger than $2 \mathrm{mo}$, we started to supplement any patient with serum phosphorus level if less than $3 \mathrm{mg} / \mathrm{dl}$.

Table I Serum Phosphorus levels During Childhood

\begin{tabular}{ll}
\hline Age & Phosphorus $(\mathbf{m g} / \mathbf{d l})$ \\
\hline $0-5$ days & $4.8-8.2$ \\
I-3 yrs & $3.8-6.5$ \\
$4-1$ I yrs & $3.7-5.6$ \\
I2- 15 yrs & $2.9-5.4$ \\
\hline
\end{tabular}

\section{Statistical analysis}

Nominal data were expressed as frequency and percentage and were compared using Chi square test. Numerical data were expressed as mean, standard deviation and range and compared using $\mathrm{T}$ test. Non-parametric data were compared using Mann Whitney test. Pearson's correlations were used to explore associations between numerical variables. A $\mathrm{p}$ value $<0.05$ was considered statistically significant. Logistic regression model was constructed using (hypophosphatemia or normal) as the dependent variable while other data were the independent (predictor) variables. Another regression model was constructed using outcome (discharged or expired) as the dependent variable while other data were the independent (predictor) variables. Forward stepwise regression method was used. In this method, the independent variables with the highest significant scores were entered in the analysis. Finally, the model tells us which independent (predictor) variables had the most significant effect on the dependent variable. For each variable in the model at each step, the loss attributed to removing that variable is computed. The more a variable contributes to the model, the larger the change in -2loglikelihood.

\section{Results}

Of the 72 patients admitted to PICU during the study period, 10 stayed less than 48 hours, 54 had been discharged and 18 died. The mean serum phosphorus level was $(3.5 \mathrm{mg} / \mathrm{dl}$ for day $1 ; 3.7 \mathrm{mg} /$ dl for day7). The mean serum phosphorus level was significantly lower in those less than 3yrs old when compared to those above $3 y r s$ old, $p<0.0001$. As shown in (Table 2), the prevalence of hypophosphatemia on day 1 was $58 \%(n=42 / 72)$. Seven percent of the patients $(n=5)$ developed hypophosphatemia during their PICU stay, and $12.5 \%(\mathrm{n}=9)$ of children who were hypophosphatemic at day 1 remained hypophosphatemic at day7, while $8 \%(n=6)$ recovered from hypophosphatemia with treatment.

Regarding the admission diagnoses of patients participating in the study, the majority were suffering of respiratory disorders as pneumonias, bronchiolitis, bronchial asthma, pneumothorax, pleural effusion and stridor. Thirty five percent $(n=25)$ of all children were mechanically ventilated. Sixty eight percent of ventilated children were hypophosphatemic ( $\mathrm{n}=17$ vs $\mathrm{n}=8$ ). Hypophosphatemic patients tended to spend more days being ventilated ( 7.0 vs 2.75 days) ( $p=$ 0.0362 ) (Table 2). Malnutrition (defined by patient's weight less than 2SD) was not a significant risk factor when compared to patient's outcome (discharged/died) $(p=0.1753)$; whereas the number of starvation days ( 4.69 vs 2.5 days; for hypophosphatemia and normophosphatemia at day7 respectively) affected the phosphorus level during the PICU stay and it was highly statistically significant ( $<0.0004)$.

Although $58 \%$ of our children were taking ranitidine and omeprazole during their PICU stay, they did not affect the serum phosphorus level. It was similar for all the drugs taken in consideration that they cause hypophosphatemia. Apart from positive culture for methicillin resistant Staphyloccocus aureus (MRSA) ( $\mathrm{p}=0.01$ ), none of the other risk factors for infection correlated significantly with hypophosphatemia including CRP and positive cultures. PIM values were significantly higher in hypophosphatemic patients (6.4) compared to normo-phosphatemics $(4.1)(\mathrm{p}=0.0237)$ (Table 3). Comparing the duration of stay at PICU, those with the normal serum phosphorus level were discharged earlier than those with hypophosphatemia (1.6 vs 2.5 days; respectively) $\mathrm{p}=0.0001$.

Patients with normal serum phosphorus level on admission (day1) had a better outcome (discharged) compared to those with hypophosphatemia (expired), although the difference was not statistically significant $(\mathrm{p}=0.2567)($ Table 4,5$)$.

Table 2 Demographic characteristics of the study population. *Others: Gastroenteritis, Guillian-Barre Syndrome, Encephalitis

\begin{tabular}{llllll}
\hline Variables & Total of Patients & Percentage of Patients & Hypo-phosphatemia & Normo-phosphatemia & PValues \\
\hline Patients Count & 72 & & 42 & 30 & 0.7885 \\
Age (mo) & & $15.08( \pm 34.19)$ & $13.1( \pm 28.38)$ & 0.0653 \\
Gender & 33 & $46 \%$ & 22 & 11 & 0.2184 \\
Female & $54 \%$ & 20 & 19 & \\
Male & & & & \\
Admission Diagnosis & & $67 \%$ & 27 & 21 & \\
Respiratory Disorders & 48 & $8 \%$ & 3 & 3 & \\
Sepsis & 6 & $4 \%$ & 1 & 2 & \\
Heart Failure & 3 & $21 \%$ & 11 & 4 & \\
Others* & 15 & $35 \%$ & 17 & 8 & 0.0362 \\
Mechanical Ventilated & 25 & & & & \\
\hline
\end{tabular}


Table 3 Risk factors of hypophosphatemia

\begin{tabular}{|c|c|c|c|c|c|}
\hline Variables & & Percentage of Patients & Hypo-phosphatemia & Normo-phosphatemia & PValues \\
\hline Malnutrition & 15 & $21 \%$ & 7 & 8 & 0.1753 \\
\hline Drugs taken & & & & & 0.5792 \\
\hline Catecholamines & 19 & $26 \%$ & I & 18 & \\
\hline Steroids & 28 & $39 \%$ & 4 & 24 & \\
\hline Diuretics & 8 & $11 \%$ & 0 & 8 & \\
\hline Ranitidine/omeprazole & 42 & $58 \%$ & 7 & 35 & \\
\hline Anticonvulsants & 14 & $19 \%$ & 3 & II & \\
\hline Culture & & & & & 0.0792 \\
\hline Not available & 27 & $38 \%$ & 13 & 14 & \\
\hline Negative & 16 & $22 \%$ & 13 & 3 & \\
\hline Positive & 29 & $40 \%$ & 16 & 13 & \\
\hline CRP & & & & & 0.274 \\
\hline Not available & 7 & $10 \%$ & 4 & 3 & \\
\hline Negative & 31 & $43 \%$ & 19 & 12 & \\
\hline Positive & 34 & $47 \%$ & 19 & 15 & \\
\hline PIM & 72 & & 42 & 30 & 0.0237 \\
\hline
\end{tabular}

NB: the not available results were considered a negative result

Table 4 Predictors of Hypophosphatemia

\begin{tabular}{llllll}
\hline Variable & & Model Log Likelihood & Change in -2 Log Likelihood & df & Sig. of the Change \\
\hline Step I & PO4atDayl & -10.974 & 9.810 & 1 & .002 \\
Step 2 & Ca & -14.209 & 20.516 & 1 & .000 \\
& PO4atDayI & -10.943 & 13.983 & .000 & 1 \\
\hline
\end{tabular}

Table 5 Survival to discharge of study population

\begin{tabular}{lllll}
\hline & $\begin{array}{l}\text { Number of } \\
\text { Patients }\end{array}$ & $\begin{array}{l}\text { Percentage of } \\
\text { Patients }\end{array}$ & $\begin{array}{l}\text { Patients with Hypo- } \\
\text { phosphatemia }\end{array}$ & $\begin{array}{l}\text { Patients with Normo- } \\
\text { phosphatemia }\end{array}$ \\
\hline Outcome & & & & $\begin{array}{l}\text { P } \\
\text { Values }\end{array}$ \\
Discharged & 54 & $75 \%$ & 29 & 25 \\
Expired & 18 & $25 \%$ & 13 & 5 \\
\hline
\end{tabular}

\section{Discussion}

The prevalence of hypophosphatemia in our prospectively studied CIC was $42 \%$; lower than the $76 \%$ reported in a retrospective study conducted in CIC by Menezes et al., ${ }^{15}$ Children younger than 3years old were more affected due to their lower body reserve compared older ones. In the present study, $56 \%$ of patients presenting with respiratory disorders were hypophosphatemic. The adding-on effect of hypophosphatemia to their respiratory problems might be attributed to the fact that hypophosphatemia is known to lead to muscle weakness and hypotonia. Fiaccadori et al., ${ }^{16}$ found that $25 \%$ of adult patients admitted to the ICU with chronic obstructive pulmonary disease were found to be hypophosphatemic.

Hypophosphatemic patients were more likely to be ventilated and to spend more days on ventilation than normo-phosphatemic patients. This might be explained by the fact that hypophosphatemia causes deficiency in the intermediary compounds for energy production, such as adenosine tri-phosphate and 2,3-diphosphoglycerate and alterations in energy metabolism, which may lead to respiratory muscle weakness and consequent worsening of respiratory insufficiency. ${ }^{5}$ The difficulty of weaning patients from mechanical ventilation is because of reduced efficiency of respiratory muscular contraction. ${ }^{17}$

In our study, none of the drugs known to deplete serum phosphorus levels as a side effect to their use (catecholamines, antacids, anticonvulsants, steroids, diuretics), showed association with hypophosphatemia. Souza de Meneses et al. ${ }^{15}$ reported same results concerning diuretics, steroids and sepsis. On the contrary, Santana e
Meneses et al. ${ }^{1}$ in their study in PICU in 2009 found that the use of dopamine was associated with hypophosphatemia and attributed this to increased urinary phosphorus excretion. Also, more than one study reported the association between hypophosphatemia and diuretics ${ }^{5}$, steroids ${ }^{13}$ and sepsis ${ }^{18}$. The absence of this association in our study can be due to the heterogeneity of the studied group, which makes each risk factor of a small sample size. Also, this can be explained by the dose and the duration of these drugs which can be not long enough to do this effect.

In contrast to the data reported in the literature, our study found no significant association with previously described factors associated with hypophosphatemia, such as malnutrition, diuretics and sepsis. Only positive cultures for MRSA showed significant negative correlation with serum phosphorus level. We hypothesized that patients with MRSA are more severely ill being hospital acquired and not covered by our first line antibiotics in the PICU which is amikacin and ampicillin/sulbactum. On the other hand, Antachopoulos et al., ${ }^{19}$ in studying acute infectious disease in pediatrics, not including CIC, demonstrated significant negative correlation between serum level of phosphate and CRP. Barak et al., ${ }^{20}$ also demonstrated that infections and sepsis were correlated with hyposhosphatemia.

Although the lack of association observed in our study between serum phosphorus level and malnutrition, hypophosphatemia was significantly affected with increased starvation days. We explain this by the fact that serum phosphorus level is affected by recent insult (starvation) rather than long standing one (malnutrition) due 
to rapid renal excretion of phosphorus. Santana e Meneses et al., failed to prove this association, and explained this by having a small sample size to detect statistical association among the two variables. Our study showed that PIM values were significantly higher in hypophosphatemic children. However, Souza de Meneses et al., ${ }^{15}$ found no significant association between PIM and hypophosphatemia.

Our results agreed with the fact that hypophosphatemia affects the length of PICU stay. This might be explained by the effect of hypophosphatemia that can trigger myocardial dysfunction, low ATP for proper respiratory muscles contraction, leading to an increased need for ventilatory support. ${ }^{17}$ In the context of the hematological system, the decline in levels of 2,3-diphosphoglycerate triggered by hypophosphatemia increases hemoglobin's affinity for oxygen, thereby causing tissue hypoxia and leading to changes in erythrocytes and leukocyte functions, hemolytic anemia, platelet dysfunction, and thrombocytopenia. ${ }^{21}$ Moreover, this study demonstrated that patients with normal serum phosphorus level on admission had better outcome (discharged rather than died). This confirmed the results of the study done by Manary et al., ${ }^{6}$ and the recommendations noted by the review done by Souza de Meneses et al., ${ }^{2}$

\section{Conclusion}

Hypophosphatemia is frequent in children admitted to PICU. It was more prevalent in those with respiratory failure, those with more starvation days and higher PIM. More length of stay and worse outcome were associated with hypophosphatemia. The association of hypophosphatemia and severity of disease needs further investigations. Our study highlights the importance of serum phosphorus level as predictive for the course of illness and outcome. More studies are needed to delineate the risk factors of admission hypophosphatemia and development of hypophosphatemia in the PICU, separately.

\section{Acknowledgments}

None.

\section{Conflicts of interest}

Author declare there are no conflicts of interest.

\section{Funding}

None.

\section{References}

1. Santana e Menses JE, Leite HP, de Carvalho WB, et al. Hypophosphatemia in critically ill children: prevalence and associated risk factors. Pediatr Crit Care Med. 2009;10(2):234-238.

2. De Menezes FS, Leite HP, Fermandez J, et al. Hypophosphatemia in critically ill children. Rev Hosp Clin Fac Med Sao Paulo. 2004; 59(5):306-311.

3. Berndt TJ, Schiavi S, Kumar R. "Phosphatonins" and the regulation of phosphorus homeostasis. Am J Physiol Renal Physiol. 2005; 289(6):F1170-F1182.
4. Miller DW, Slovis CM. Hypophosphatemia in the emergency department therapeutics. Am J Emerg Med. 2000;18(4):457-461.

5. Subramanian R, Khardori R. Severe hypophosphatemia Pathophysiologic implications, clinical presentations, and treatment. Medicine (Baltimore). 2000;79(1):1-8.

6. Manary MJ, Hart CA, Whyte MP. Severe hypophosphatemia in children with kwashiorkor is associated with increased mortality. J Pediatr. 1998;133(6):789-791.

7. Heames RM, Cope RA. Hypophosphatemia causing profound cardiac failure after cardiac surgery. Anaesthesia. 2006;61(12):1211-1213.

8. Gassbeek A, Meinders AE. Hypophosphatemia: an update on its etiology and treatment. Am J Med. 2005;118(10):1094-1101.

9. Afzal NA, Addai S, Fagbemi A, et al. Refeeding syndrome with enteral nutrition in children: a case report, literature review and clinical guidelines. Clin Nutr. 2002;21(6):515-520.

10. Worley G, Claerhout SJ, Combs SP. Hypophosphatemia in malnourished children during refeeding. Clin Pediatr. 1998;37(6):347-352.

11. Landenberg PV, Shoenfeld Y. New approaches in the diagnosis of sepsis. Isr Med Assoc J. 2001;3(6):439-442.

12. Shoenfeld Y, Hager S, Berliner S, et al. Hypophosphatemia as diagnostic aid in sepsis. N Y State J Med. 1982;38(2):163-165.

13. Thomas C, Fourrier F. Hypophosphorémies en réanimation. Réanimation. 2003;12:280-287.

14. Greenbaum LA. Electrolyte and acid- base disorders. In: Kligman RM et al. (Eds.), Nelson Textbook of Pediatrics ( $18^{\text {th }}$ edn), WB Saunders Company, USA. 2008. p.288.

15. De Menezes FS, Leite HP, Fernandez J, et al. Hypophosphatemia in children hospitalized within an intensive care unit. $J$ Intensive Care Med. 2006;21(4):235-239.

16. Fiaccadori E, Coffrini E, Fracchia C, et al. Hypophosphatemia and phosphorus depletion in respiratory and peripheral muscles of patients with respiratory failure due to COPD. Chest. 1994;105(5):1392-1398.

17. Aubier M, Murciano D, Lecogguic Y, et al. Effect of hypophosphatemia on diaphragmatic contractility in patients with acute respiratory failure. N Engl J Med. 1985;313(7):420-424.

18. Zazzo JF, Troche G, Ruel P, et al. High incidence of hypophosphatemia in surgical intensive care patients: efficacy of phosphorus therapy on myocardial function. Intensive Care Med. 1995;21(10):826-831.

19. Antachopoulos C, Margeli A, Giannaki M, et al. Transient hypophosphatemia associated with acute infectious disease in pediatric patients. Scand J Infect Dis. 2002;34(11):836-839.

20. Barak V, Schwartz A, Kalickman I, et al. Prevalence of hypophosphatemia in sepsis and infection: the role of cytokines. Am J Med. 1998;104(1):40-47.

21. Solomon SM, Kirby DF. The Refeeding Syndrome: A Review. JPEN J Parenter Enteral Nutr. 1990;14(1):90-97. 